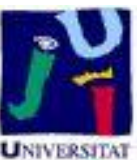

Título artículo / Títol article: Elucidating Operating Modes of BulkHeterojunction Solar Cells from Impedance Spectroscopy Analysis

Autores / Autors

García Belmonte, Germà ; Guerrero Castillejo, Antonio ; Bisquert, Juan

Revista:

The Journal of Physical Chemistry Letters

Versión / Versió:

Post-print

Cita bibliográfica / Cita bibliogràfica (ISO 690):
GARCIA-BELMONTE, Germà; GUERRERO, Antonio; BISQUERT, Juan. Elucidating operating modes of bulk-heterojunction solar cells from impedance spectroscopy analysis. The Journal of Physical Chemistry Letters, 2013, vol. 4, no 6, p. 877-886.

url Repositori UJI:

http://hdl.handle.net/10234/89431 


\title{
Elucidating Operating Modes of Bulk-Heterojunction Solar Cells from Impedance Spectroscopy Analysis
}

\author{
Germà Garcia-Belmonte*, Antonio Guerrero, and Juan Bisquert \\ Photovoltaic and Optoelectronic Devices Group, Departament de Física, Universitat \\ Jaume I, ES-12071 Castelló, Spain
}

\begin{abstract}
We discuss the progress and challenges in the application of impedance spectroscopy analysis to determine key processes and parameters in organic bulk-heterojunction solar cells. When carrier transport or outer interface extraction do not severely influence the solar cell performance, a simple method to quantify the open-circuit voltage loss caused by the kinetics of charge carrier recombination is provided, based on the determination of chemical capacitance and recombination resistance. This easily allows distinguishing between energetic and kinetic effects on photovoltage, and establishes a benchmark for the performance comparison of a set of different cells. A brief discussion of impedance analysis in the much less studied case of collection-limited solar cells is introduced.
\end{abstract}

keywords: Organic Electronics, Photovoltaic Devices, Carrier Recombination, Impedance Spectroscopy

*Corresponding author: G. Garcia-Belmonte, e-mail: garciag@,fca.uji.es, tel.: +34964 387548, fax: +34964729218 
Organic photovoltaic technology can potentially reduce production costs of solar energy by adopting cheaper printing technologies. A considerable effort has very recently led to achieve power conversion efficiencies near $10 \%$ in the case of bulk-heterojunction (BHJ) solar cell structures. ${ }^{1}$ Improvement in solar cell efficiency partially relies upon achieving higher open-circuit voltages $V_{\text {oc }}$ by a reduction of the impact of charge carrier recombination losses. ${ }^{2}$ Carrier recombination is understood as a charge transfer event which involves occupied acceptor fullerene molecules and unoccupied donor polymer chains. By inhibiting the internal recombination flux to some extent, the amount of charge carriers available to the photovoltaic operation is enhanced, yielding accordingly larger $V_{\mathrm{oc}}$. Carrier recombination flux is known to be determined by both material energetics as well as charge transfer kinetics. However the sole analysis of the current-voltage $j-V$ dc curves does not permit a clear distinction between energetic and kinetic influences on the achieved $V_{\mathrm{oc}}$. Only frequency-resolved (impedance spectropscopy, IS) ${ }^{3}$ and time-resolved (transient) ${ }^{4}$ electrical techniques directly face that distinction, allowing for a simultaneous determination of energetic and kinetic effects. We will focus here on the first technique to determine voltage losses caused by the kinetics of recombination from resistance and capacitance measurements in a simple way. Recently the application of impedance spectroscopy to analyze recombination properties as well as other key aspects of the operation of bulk-heterojunction organic solar cells has been increasingly used. We provide in this Perspective a review of recent progress in these methods as well as an outline of the major problems remaining in this field.

We proposed in 2008 an initial method ${ }^{5}$ to address a meaningful interpretation of the parameters extracted from IS of BHJ solar cells. Since then many groups ${ }^{6}$ have adopted IS as a useful tool for organic solar cell characterization, and more importantly, towards a determination of operating mechanisms. IS responses of organic solar cells are considerably simplified in those cases in which carrier transport or outer interface extraction do not severely limit the cell performance. The interpretation of capacitive and resistive elements under these conditions is now well-established in terms of distributions of bandgap density-of-states (DOS), and carrier recombination currents. Reconstruction of $j-V$ curves from IS-extracted parameters is in agreement with an illumination intensity-independent recombination current, and a constant photocurrent which is not altered by the applied voltage. These mechanisms are visible within the low-frequency part of the impedance spectra. The central relationship between such

Published in Journal of Physical Chemistry Letters 2013, 4, 877-886 
circuit elements and the achievable open-circuit voltage is addressed here in detail, and forms the main part of this paper.

On the other hand, our current knowledge about IS analysis when carrier transport or extraction dominate the solar cell performance is much more scarce. Only a few works have treated the repercussion of the series resistance, or the effect of oxide interlayers on the overall solar cell behavior. The analyses proposed until now are by far much speculative, and more work is necessary. Particularly interesting is the comprehension of the mechanisms limiting charge collection, consequently reducing measured photocurrent at short circuit. We provide here some guiding remarks about these topics in the final section.

Electronic density-of-states and open-circuit voltage. The net effect of incoming photon energy upon organic materials comprising the blend of bulk-heterojunction solar cells is the generation of charge carriers. The resulting photovoltage appears as a consequence of the splitting of the separate Fermi levels promoted by the increase in the DOS occupancy $^{7}$

$$
q V_{F}=E_{F n}-E_{F p} .
$$

The main question that must be addressed is the connection of carrier distribution to the variation of the Fermi levels. In samples with poor intrinsic conductivity those carriers are used to develop space charge regions and modify electrical fields that connect directly to the photovoltage. On the other hand, in devices in which the active layer is doped and contains an appreciable amount of majority carriers, the role of electrical fields and space charge regions can be of marginal significance. This latter case is what we have found in many cases of interest. ${ }^{2 b}$ It follows that we can regard carrier distribution as largely homogeneous. But then, another aspect has a strong influence on the build-up of the photovoltage in eq 1, and this is the energy disorder. Materials employed in organic photovoltaic devices usually produce complex morphologies, hence the connection between the actual DOS and statistical properties of carriers have a great influence on determining the Fermi levels of electrons and holes. The actual position of the electron and hole Fermi levels within the existing DOS poses strong constraints on the phenomenology of operation of organic solar cells, determining the variation of several cell parameters on temperature and light-intensity. ${ }^{7-}$ ${ }^{8}$ The Fermi level splitting view of eq 1 is richer than simpler approaches based on 
monoenergetic considerations as it incorporates not only the influence of energetic disorder but also the inherent contribution of entropic effects. On the contrary adopting solely the difference between the acceptor lowest-unoccupied molecular orbital LUMO and donor highest-occupied molecular orbital HOMO levels one might just expect a rough $V_{\text {oc }}$ estimation that largely differs from values actually measured.

Another important issue for the assessment of photovoltage in BHJ solar cells that has not received sufficient attention is the current experimental methods to state the energy levels of the organic materials. Energetic position of relevant HOMO and LUMO levels is commonly derived from cyclic voltammetry CV and ultraviolet photoelectron spectroscopy UPS experiments. It is however recognized that organic layers prepared to be tested using CV and UPS often exhibit morphological and even compositional differences from those making up the active layer in complete solar devices. Moreover these techniques are sensitive to charge densities that exceed by several orders of magnitude that found in organic solar cells under usual operation conditions $\sim 10^{15}-10^{17} \mathrm{~cm}^{-3}$. Therefore inferences about active layer energetics extracted from CV and UPS experiments are only approximate, and can be used in some cases only as an upper limit orientation.

The actual energy landscape exhibited by organic compounds comprising the active layer in complete cells is inherently complex. This is obviously the case of donor and acceptor materials forming a blend of intricate morphology. Therefore the tendency for carrier localization found in organic compounds is enhanced by the presence of energetic disorder, which is the result of structural inhomogeneities and chemical impurities. ${ }^{9}$ It is then expected featured DOS distributions entering the effective bandgap for both donor and acceptor materials.

Accessing relevant DOS in blended materials forming the active layer of real devices is not a simple task. A useful technique consists of measuring the capacitance exhibited by complete solar cells operating within the power-generating quadrant. Capacitance is obtained as a response to a small-amplitude perturbation of a given steady-state as described in many of our previous works, ${ }^{10}$ although it is possible to address the same quantity by charge extraction techniques. ${ }^{4}$ Under certain experimental conditions the chemical capacitance ${ }^{11}$ is extracted which responds to the modulation of quasi-free charge carrier density with respect to the voltage (Fermi level splitting).

We have observed in a variety of polymer/fullerene blends that holes form a set of majority carriers in the active blend so that the hole Fermi level is basically stable. This

Published in Journal of Physical Chemistry Letters 2013, 4, 877-886 
is because polymer films commonly undergo unintended oxidation upon exposure to the air or moisture, ${ }^{12}$ that ultimately creates a background density of mobile holes $p_{0}$. This is confirmed by independent measurements that evidence the existence of a significant amount of background carriers because of the polymer doping caused by structural and chemical defects. ${ }^{13}$ The amount of excess, photogenerated holes $\Delta p=p-p_{0}$ for usual illumination intensities around 1 sun, lies within the order of magnitude of the density of dopants $p_{0} \approx \Delta p=10^{16}-10^{17} \mathrm{~cm}^{-3}{ }^{2 a}$ Fullerene molecules are much more stable in air without significant reduction. Therefore due to the $p$-doping character of the polymer the downward shift of $E_{F p}$ by effect of illumination or applied voltage should be restricted within a few $k_{\mathrm{B}} T$ (thermal energy), as $E_{F 0}-E_{F p} \approx k_{\mathrm{B}} T \ln \left(p / p_{0}\right)$. Therefore the shift of Fermi levels originated by excess carriers mainly affects the electron level $E_{F n}$ which then screens a large portion of the effective band gap (Figure 1). On the contrary the hole Fermi level $E_{F p}$ makes up a sort of energy reference. It should be remarked that these assumptions do not hold in the case of insulating or very poorly doped blends in which the notion of a minority carrier and the interpretation of the capacitance required a separate analysis, that is beyond the scope of this Perspective.

In the general mentioned conditions, the device capacitance is governed by a chemical capacitance due to the excess carriers $C_{\mu},{ }^{3,14}$ which is related to the change in the occupancy of fullerene LUMO states by electrons, as follows

$$
C_{\mu}=q^{2} L \frac{d n}{d E_{F n}}
$$

Here the capacitance is given per unit area, and $n$ corresponds to the electron concentration, $q$ stands for the elementary charge, and $L$ is the active layer thickness. An increase of $C_{\mu}$ is expected at forward bias as the DOS occupancy progresses. The chemical capacitance is in fact an approximate replica of the electron DOS as $C_{\mu}=q^{2} \operatorname{Lg}_{n}\left(V_{F}\right) .^{3,15}$ This last correspondence is strictly valid at zero-temperature when the Fermi-Dirac function converges to the step function. 

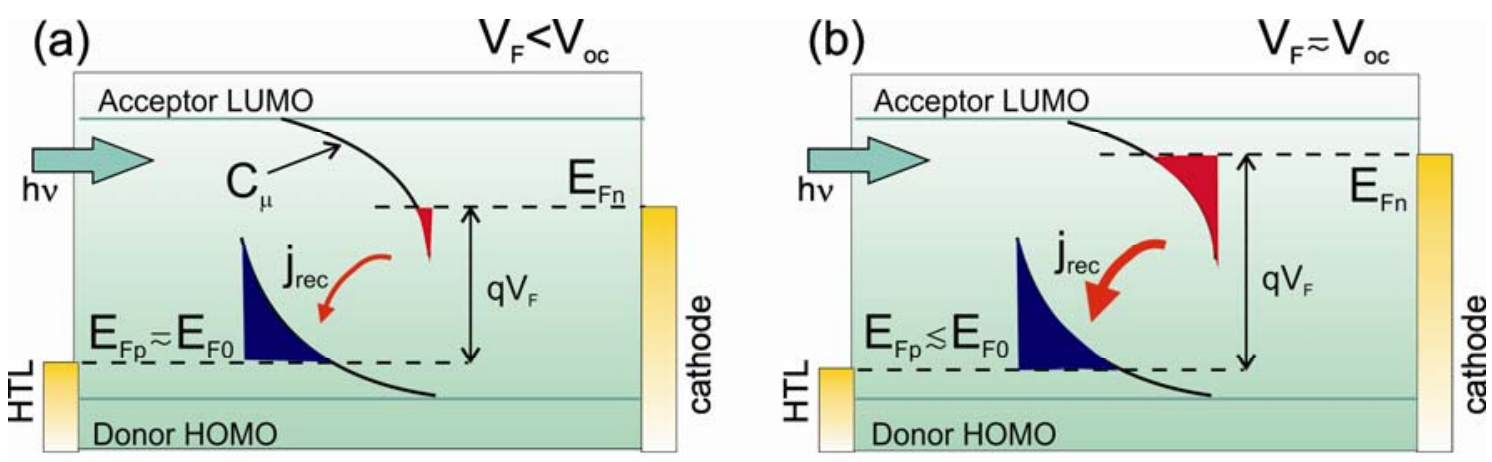

(c)

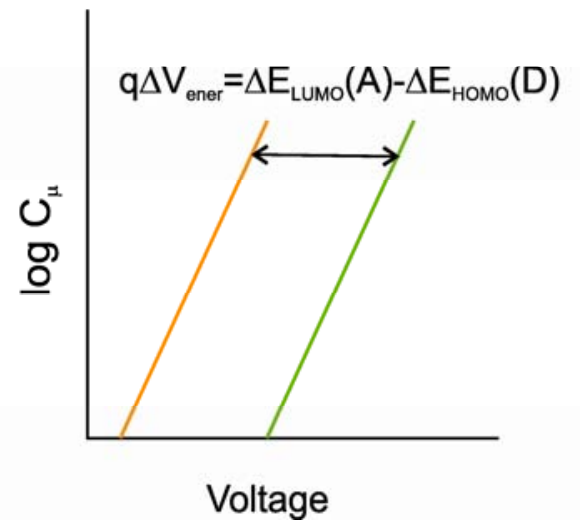

(d)

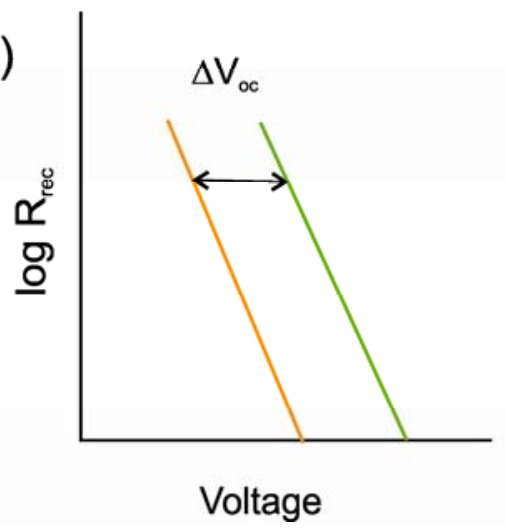

Figure 1. Mechanisms governing the Fermi level location within the effective bandgap established between donor HOMO and acceptor LUMO levels. For simplicity DOS occupation is limited to the Fermi level (shaded zones). The separate Fermi levels determine the photovoltage as $q V_{F}=E_{F n}-E_{F p}$. (a) For forward voltages below $V_{\mathrm{oc}}$ the hole Fermi level practically equals the equilibrium value $E_{F p} \approx E_{F 0}$ stated by the background carrier density. Only slight occupancy is expected for electron DOS. The measured chemical capacitance $C_{\mu}$ follows the fullerene LUMO occupation. (b) Larger voltages approaching $V_{\text {oc }}$ promote electrons to acceptors states, while hole Fermi level undergoes small downward shift. Charge transfer events between occupied acceptor fullerenes and unoccupied donor molecules are enhanced giving rise to larger recombination current. (c) When two different cells are compared, the $C_{\mu}$ voltage shift correlates to the effective bandgap offset as $q \Delta V_{\text {ener }}=\Delta E_{\mathrm{LUMO}}(A)-\Delta E_{\mathrm{HOMO}}(D)$. (d) Direct relationship between the voltage shift in the recombination resistance for two different solar cells, and the open-circuit voltage offset.

A number of chemical capacitance measurements extracted for a variety of polymer/fullerene blends is shown in Figure 2. These data correspond to our recent publications on this subject, and detailed information about compounds, structures, and 
processing conditions is included as Supporting Information. Some new data are also included. As a general trend it is found a rise in capacitance values at large voltages approaching $V_{\mathrm{oc}}$. This capacitance, which is in excess of geometrical values responding at low voltages, is related to the increase in the DOS occupancy as introduced previously. Generally we have found an exponential increment of the type $C_{\mu}=C_{0} \exp \left(\alpha q V_{F} / k_{\mathrm{B}} T\right)$ with $\alpha \approx 0.3-0.4$ according to an exponential bandgap DOS. The occupancy of bandgap states is modulated by a parameter $\alpha$, which accounts for the characteristic energy of the DOS $\alpha=T / T_{0}$, being $T_{0}$ the characteristic temperature of the exponential distribution $g(E) \propto \exp \left(E / k_{\mathrm{B}} T_{0}\right) \propto \exp \left(\alpha E / k_{\mathrm{B}} T\right)$. At low voltages the measured capacitance approaches the geometrical, dielectric value. These trends are clearly visible in Figure 2 for several polymer: [6,6]-phenyl $\mathrm{C}_{61}$-butyric acid methyl ester (PCBM) combinations. ${ }^{16}$

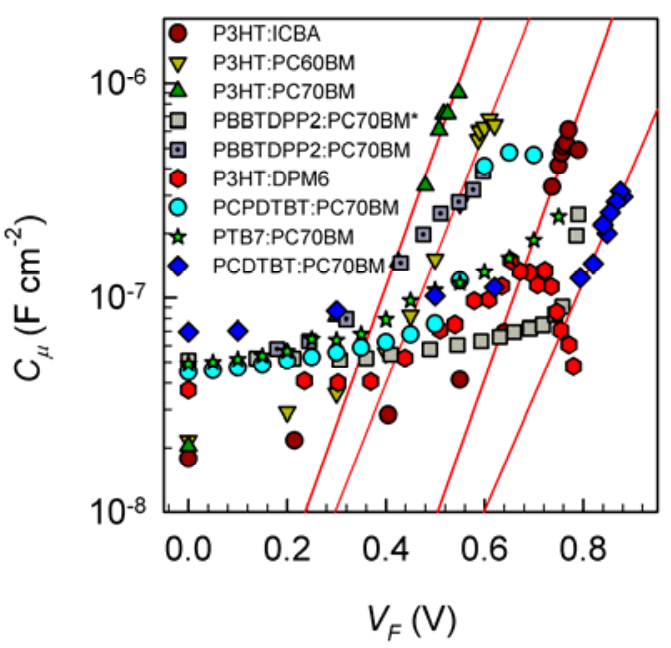

Figure 2. Capacitance-voltage extracted from impedance analysis for a variety of different materials forming the blend of bulk-heterojunction solar cells. Screened fullerenes comprise molecules with different electron affinity. Polymers of wide and low bandgap, and different ionization energy are analyzed. As a general trend a rise in capacitance is observed in excess of dielectric values as $C_{\mu}=C_{0} \exp \left(\alpha q V_{F} / k_{\mathrm{B}} T\right)$. Straight lines correspond to the exponential behavior when P3HT and PCDTBT are used as donor polymer. The case of low-bandgap DPP-based cells processed from either $O$ dichlorobenzene or chloroform (*) solvents is included. 
Indeed straight lines in Figure 2 correspond to exponential fits in which only high voltage capacitance values of poly(3-hexylthiophene) (P3HT) blended with PCBM and Indene- $\mathrm{C}_{60}$ bisadduct (ICBA) fullerene molecules are considered. As stated in eq 1 the output voltage depends on the splitting of the separate Fermi levels. As commented before the hole Fermi level only experiences a slight downshift in comparison to the large offset encountered for the electron Fermi level (Figure 1). This implies that the hole Fermi level can be used as an energy reference because $E_{F p} \approx E_{F 0}$, being $E_{F 0}$ the equilibrium value. When the polymer doping attains comparable levels in a number of samples it is feasible to correlate the voltage shift in the capacitance plot (Figure 2) directly to the offset in the fullerene LUMO level, as expected when the bandgap DOS position follows the electron affinity energies. ${ }^{16}$ The effect in the polymer HOMO offset is also visible in Figure 2 as a voltage shift when poly-carbazole (PCDTBT) is used as donor system in comparison to P3HT-based cells. In other cases as the same fullerene is used in inverted and standard solar cells, the $p$-doping level can be derived from the voltage shift. ${ }^{17}$

In those cases in which the capacitance exhibits an exponential behavior it is even feasible to estimate the energy shift induced by material or processing changes. We interpret the voltage shift in the capacitance vs. voltage plot in terms of variations of the polymer HOMO and fullerene LUMO energies as $q \Delta V_{\text {ener }}=\Delta E_{\mathrm{LUMO}}(A)-\Delta E_{\mathrm{HOMO}}(D)$, when a specific solar cell is taken as a reference. This is schematically illustrated in Figure 1(c), and listed in Table I for a variety of solar cells.

It is known that specific processing methods determine to a large extent the effective energy level distribution. For example the use of different solvents considerably affects the solar cell performance and operation. It has been reported that low-bandgap diketopyrrolopyrrole-based PBBTDPP2 polymer processed in o-dichlorobenzene is partly aggregated and shows two consecutive onsets of oxidation for aggregated and fully dissolved chains which are $0.27 \mathrm{eV}$ apart. ${ }^{18}$ Polymer aggregation then stabilizes energy levels by moving them upward causing both the observed red shift and partially the offset in $V_{\text {oc }}$. This is observed in Figure 2 as a capacitance shift between DPP cells processed either with $o$-dichlorobenzene or chloroform solvents. ${ }^{19}$

Other chemical capacitance measurements have revealed a different behavior. This was the case of 4,40-dihexyloxydiphenylmethano-[60] fullerene (DPM6) fullerene molecules blended with P3HT polymer. ${ }^{15}$ Contrary to PCBM acceptor, DPM6 exhibits 
low aggregation so that we observed that DPM6-based solar cells reach higher $V_{\text {oc }}$ values because of a smaller intermediate DOS that lies below the upper LUMO manifold of the fullerene. Remarkably, the existence of larger DOS in the case of PCBM has the detrimental consequence of keeping the Fermi level within the tail of the distribution, therefore limiting the rise of the output $V_{\mathrm{oc}}$. Because the main difference between PCBM and DPM6 is observed to be the less crystalline nature of the latter, we suggested that the origin of the electronic intermediate bands lies in the existence of pure fullerene (PCBM) crystals with reduced energy gaps. As shown in Figure 2 capacitance of DPM6-based solar cells exhibits a complete, Gaussian-like band of electronic states of lower density related to a small number of available sites. We remark that the chemical capacitance measurement is able to directly monitor the energy position of the relevant DOS actually influencing the final value of the open-circuit voltage in complete cells. Capacitive measurements allow distinguishing between electronic bands from site tails, and their relative position within the effective bandgap.

A way to understand the drastic effect of energetic disorder represented by DOS into the output $V_{\text {oc }}$ is by means of device simulations. In the ideal case in which transport mechanisms do not severely limits the solar cell performance eq 1 can be used as a starting point to derive the achievable $V_{\text {oc }}$. This was addressed in previous papers assuming a Gaussian DOS both for electrons at acceptor LUMO manifold as well as holes occupying donor HOMO levels. The crucial parameter here is the DOS width $\sigma$ accounting for the energetic disorder degree. We demonstrated that for weak disorder $\sigma \approx 50 \mathrm{meV}$ and high temperature regime eq 1 gives rise to the next expression in terms of the carrier density as ${ }^{7}$

$$
q V_{\mathrm{oc}}=E_{g}^{\sigma}-k_{\mathrm{B}} T \ln \left(\frac{N_{\mathrm{LUMO}} N_{\mathrm{HOMO}}}{n p}\right)
$$

where $n(p)$ stand for the electron (hole) concentration. $N_{\mathrm{LUMO}}$, and $N_{\mathrm{HOMO}}$ are the acceptor LUMO, and HOMO total density, and

$$
E_{g}^{\sigma}=E_{g}^{0}-\frac{\sigma_{n}{ }^{2}+\sigma_{p}{ }^{2}}{2 k_{\mathrm{B}} T}
$$

Here $E_{g}^{0}=E_{\mathrm{LUMO}}(\mathrm{A})-E_{\mathrm{HOMO}}(\mathrm{D})$ corresponds to the effective energy gap. Equation 4 contains the term related to the electron and hole equilibration energies in the disordered DOS as $\sigma^{2} / k_{\mathrm{B}} T$ with the net effect of reducing the effective bandgap from $E_{g}^{0}$ to $E_{g}^{\sigma}$. For large disorder values $(\sigma \geq 100 \mathrm{meV})$ usually encountered in real 
devices the detrimental influence on $V_{\text {oc }}$ is severe. $^{20}$

Kinetics of carrier recombination. The parameterization of $j-V$ characteristics exhibited by organic solar cells is usually written in terms of an exponential dependence as

$$
j=j_{0}\left[\exp \left(\beta \frac{V_{F}}{k_{\mathrm{B}} T / q}\right)-1\right]-j_{\mathrm{ph}}
$$

where $j_{0}$ stands for the saturation current, $j_{\mathrm{ph}}$ accounts for the photocurrent, and $\beta$ corresponds to the inverse of the diode ideality factor. In those cases in which transport and extraction processes do not limit the cell operation eq 5 can be entirely interpreted in terms of the carrier recombination flux occurring in the active layer. This is labeled as the $\beta$-recombination model since the exponential behavior relates to the recombination current,

$$
j_{\text {rec }}=j_{0}\left[\exp \left(\beta \frac{V_{F}}{k_{\mathrm{B}} T / q}\right)-1\right]
$$

which relates to the set of charge transfer events between occupied acceptor LUMO and unoccupied donor HOMO levels. We have addressed recently the phenomenology of recombination expressed in eq 6 by looking at the recombination resistance using IS measurements. ${ }^{10}$ The recombination resistance is readily obtained from the impedance measurement at low frequencies. This quantity corresponds to the derivative of the recombination current as

$$
R_{\mathrm{rec}}=\frac{1}{L}\left(\frac{d j_{\mathrm{rec}}}{d V_{F}}\right)^{-1}
$$

$R_{\text {rec }}$ contains a great deal of information on recombination flux. Recombination properties can be discussed by observing the variation of $R_{\text {rec }}$ with voltage or by comparing samples with different morphologies and energetics. We have observed that for P3HT:PCBM solar cells recombination and photogeneration currents are separated terms, i.e. recombination current results independent of the illumination level, and photocurrent is voltage-independent. ${ }^{21}$ Such a separation of summands in eq 5 occurs as far as charge transport does not severely limit the extraction of photogenerated carrier. This is usually the case of thin active layer films.

A set of recombination resistance measurements for a variety of polymer/fullerene 
blends is shown in Figure 3. We have generally observed an exponential dependence at forward voltages approaching $V_{\text {oc }}$ of the type $R_{\text {rec }}=R_{0} \exp \left(-\beta q V_{F} / k_{\mathrm{B}} T\right)$. This is in good accordance with eq 6 and the recombination resistance definition in eq 7. A straightforward estimation of $\beta$ is obtained that results in $\beta \approx 0.6-0.7$. We notice that the saturation current $j_{0}$ correlates directly to the recombination resistance prefactor as

$$
j_{0}=\frac{k_{\mathrm{B}} T}{\beta L R_{0}} .
$$

Equation 8 is a useful way to determine $j_{0}$ avoiding the disturbing effect of leakage currents on the $j-V$ curves. At lower voltages $R_{\text {rec }}$ saturates to a value that might be either related to a change in the microscopic recombination mechanism, or more likely to the shunt resistance dominating as a consequence of unavoidable leakage currents.

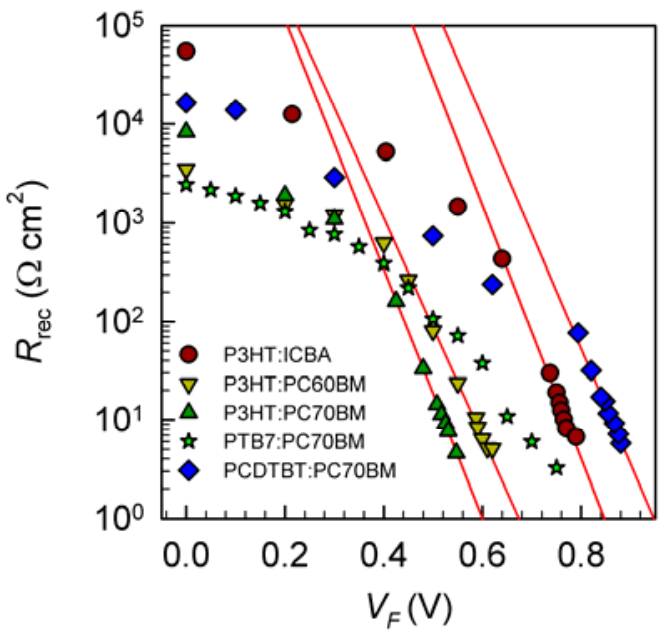

Figure 3. Recombination resistance versus voltage as derived from impedance spectroscopy analysis. A set of different responses is shown which exhibit an exponential behavior as $R_{\mathrm{rec}}=R_{0} \exp \left(-\beta q V_{F} / k_{\mathrm{B}} T\right)$.

At $V_{\text {oc }}$ the saturation current is largely exceeded by the exponential term in eq 6. By assuming a voltage-independent photocurrent $j_{\mathrm{ph}}=j_{\mathrm{sc}}$, and eq 8 one readily arrives at

$$
V_{\mathrm{oc}}=\frac{k_{\mathrm{B}} T}{q \beta} \ln \left(\frac{j_{\mathrm{sc}}}{j_{0}}\right)=\frac{k_{\mathrm{B}} T}{q \beta} \ln \left(\frac{R_{0} L \beta j_{\mathrm{sc}}}{k_{\mathrm{B}} T}\right) .
$$


This last correlation has been recently suggested for the case of dye-sensitized solar cells. $^{22}$ From eq 9 it is derived that the voltage shift encountered in the recombination resistance plot of Figure 3 directly correlates to the $V_{\text {oc }}$ offset in those cases in which $\beta$ parameter exhibits similar values. The procedure is illustrated in Figure 1(d), and corroborated in Table I for a variety of polymer/fullerene combinations of different ionization energy and electron affinity values. Differences in $\Delta V_{\mathrm{oc}}$ as derived from $j-V$ curves and that extracted from the voltage shift in the $R_{\text {rec }}$ plot are within the experimental error.

We move now to explain how impedance measurements contain the essential ingredients that allow us to separate kinetic from energetic effects on $V_{\text {oc }}$. A key parameter to understand the kinetics of charge carrier recombination in solar cells is the saturation current $j_{0} \cdot{ }^{23}$ It is known that $j_{0}$ establishes the time scale of recombination through the combined effect of the charge transfer energetics and kinetics. As suggested for inorganic semiconductors, ${ }^{23 a}$ one can propose a separation of the form

$$
j_{0}=j_{0 k} \exp \left(-\frac{\beta E_{g}}{k_{\mathrm{B}} T}\right)
$$

An expression that explicitly separates kinetic terms represented by the prefactor $j_{0 k}$ from energetic contributions stated through bandgap energy $E_{g}$. In inorganic materials $E_{g}$ is a single function of the semiconductor. However, in organic blends the effective gap $E_{g}^{0}=E_{\mathrm{LUMO}}(\mathrm{A})-E_{\mathrm{HOMO}}(\mathrm{D})$ is a function of the relative energetics of the blend components. Therefore assessing the influence of energetics on $j_{0}$ is a matter that requires careful investigation. Nevertheless, our approach permits evaluating $j_{0 k}$ directly from the impedance analysis rather than from the $j-V$ characteristic usually masked by leakage currents at low bias voltages. Equation 8 and fittings of the recombination resistance in Figure 3 allow calculating $j_{0}$ (see Table 1). Blends with larger effective gap yields lower $j_{0}$ values as expected. By adopting the simple form of eq 10 , it is feasible to determine $j_{0 k}$ taking into account the effective gap as derived from the capacitance voltage shift (see below). It is observed in Table I that $j_{0 k}$ lies within the range of $10^{3}-10^{4} \mathrm{~A} \mathrm{~cm}^{-2}$.

It should be remarked that when comparing a set of photovoltaic responses yielded by different cells, the simple inspection of the $j-V$ curves does not allow for a separate quantification of kinetic and energetic effects on the attained $V_{\text {oc }}$. As previously introduced the energetic shift is directly determined from the voltage offset 
of the capacitance response of two cells. Therefore the separation of kinetic from energetic effects on $V_{\mathrm{oc}}$ is accomplished as

$$
\Delta V_{\mathrm{oc}}=\Delta V_{\text {ener }}+\Delta V_{\text {kin }}
$$

Equation 11 explicitly states that the kinetic contribution is accessible from the difference between capacitance and resistance voltage offsets. According to eq 9 there is in addition a small correction resulting from the difference in $j_{\mathrm{sc}}$ as $k_{\mathrm{B}} T / q \beta \ln \left(j_{\mathrm{sc}} / j_{\mathrm{sc}}^{\prime}\right)$. However differences of a factor two in $j_{\mathrm{sc}}$ introduce a minor $V_{\mathrm{oc}}$ offset of only $17 \mathrm{mV}$ at room temperature. In Table 1 it is summarized the calculation of the kinetic loss as a reduction in $V_{\text {oc }}$ for a set of different solar cells. When $\mathrm{P} 3 \mathrm{HT}: \mathrm{PC}_{60} \mathrm{BM}$ is taken as a reference system it is observed that $\Delta V_{\text {kin }}$ term in eq 11 results always negative, thus indicating that $\mathrm{P} 3 \mathrm{HT}: \mathrm{PC}_{60} \mathrm{BM}$ exhibits the slowest kinetics of recombination. As recently shown, a variation of the fullerene electron affinity has only a minor effect on the recombination kinetics, as corroborated from the small ( $\Delta V_{\text {kin }} \sim 20 \mathrm{mV}$ ) loss encountered. ${ }^{16}$ PCDTBT shows larger voltage loss owed to higher recombination at 1 sun irradiation intensity. By examining Table 1 one can infer that the main factor determining $V_{\mathrm{oc}}$ variations among cells is differences in material energetics (stated by $\Delta V_{\text {ener }}$ ), being the kinetic contribution of secondary repercussion.

At this point we recognize that more experimental data is needed to progress in the comprehension of the recombination kinetics. We speculate here that interactions at molecular level finally determine the recombination process. This obviously points to the role that morphology plays in establishing the recombination time scale (related to the saturation current prefactor $j_{0 k}$ ). Hence processing conditions and materials properties as compound solubility, purity, molecular weight, annealing process, or additives used might have an influence. Particularly relevant seems to be the packaging between fullerene molecules and polymer chains. ${ }^{24}$

The analysis previously proposed is based on a straightforward measurement of capacitive and resistive elements given by IS. It should be stressed that the method has the advantage of an extreme simplicity, and does not assume any microscopic model for the charge transfer event responsible for the carrier recombination process. Nevertheless IS is able to capture essential contributions to the open-circuit voltage in a manner easily implementable into industrial processing lines, and establishes a benchmark for the performance comparison of a set of different cells. 
Table 1. Open-circuit voltage shift extracted from $j-V$ curves $(*)$ measured under $100 \mathrm{~mW} \mathrm{~cm}^{-2}$ simulated AM1.5 sunlight, in comparison from that calculated from $R_{\text {rec }}$ versus voltage plot (Figure 3). $\Delta V_{\text {ener }}$ energetic contribution to $\Delta V_{\mathrm{oc}}$ as derived by analyzing $C_{\mu}$ voltage shift in Figure 2. Calculation of the kinetic loss in accordance to $\Delta V_{\mathrm{oc}}=\Delta V_{\text {ener }}+\Delta V_{\text {kin }}$. In all these cases $\mathrm{P} 3 \mathrm{HT}: \mathrm{PC}_{60} \mathrm{BM}$ is taken as a reference system. Saturation current $j_{0}$ and kinetic prefactor $j_{0 k}$ calculated from eq 8 and eq 10 .

\begin{tabular}{|c|c|c|c|c|c|c|c|}
\hline blend & $\begin{array}{c}V_{\mathrm{oc}} \\
(\mathrm{mV})\end{array}$ & $\begin{array}{l}\Delta V_{\mathrm{oc}}^{*} \\
(\mathrm{mV})\end{array}$ & $\begin{array}{l}\Delta V_{\mathrm{oc}} \\
(\mathrm{mV})\end{array}$ & $\begin{array}{c}\Delta V_{\text {ener }} \\
(\mathrm{mV})\end{array}$ & $\begin{array}{l}\Delta V_{\text {kin }} \\
(\mathrm{mV})\end{array}$ & $\begin{array}{l}j_{0} \\
\left(\mathrm{~A} \mathrm{~cm}^{-2}\right)\end{array}$ & $\begin{array}{l}j_{0 k} \\
\left(\mathrm{~A} \mathrm{~cm}^{-2}\right)\end{array}$ \\
\hline P3HT:PC 60 BM & 620 & - & - & - & _ & $1.2 \times 10^{-9}$ & $2.2 \times 10^{3}$ \\
\hline P3HT:PC 70 BM & 560 & -60 & -70 & -50 & -20 & $8.7 \times 10^{-10}$ & $1.7 \times 10^{4}$ \\
\hline P3HT:ICBA & 800 & 180 & 175 & 200 & -25 & $4.2 \times 10^{-13}$ & $2.3 \times 10^{4}$ \\
\hline PCDTBT:PC ${ }_{70} \mathrm{BM}$ & 845 & 225 & 240 & 300 & -60 & $3.0 \times 10^{-13}$ & $7.4 \times 10^{3}$ \\
\hline
\end{tabular}

Transport and outer contact mechanisms. The analysis of solar cells in terms of eq 5 relies upon the identification of the applied voltage with the Fermi level splitting of eq 1. As mentioned before this identification assumes a position-independent value for the electron and hole Fermi levels which easily connect to the outer voltage without substantial gradients or steps at contacts. This situation occurs as far as the applied voltage univocally establishes the carrier density (Fermi level) at any internal point regardless the intensity of the incoming light. ${ }^{21}$ However carrier transport and contact resistances might influence the operating mode breaking the electronic reciprocity between voltage at contacts and internal carrier density. If the extraction of carriers demands a significant gradient of their concentration, then obviously the local concentration depends on the illumination level.

When transport and contact effects yet do not severely limit the device operation a useful generalization of eq 5 is based on the inclusion of series and shunt resistances as 


$$
j=\frac{1}{1+R_{\mathrm{s}} / R_{\mathrm{p}}}\left\{j_{0}\left[\exp \left(\beta \frac{V_{\mathrm{app}}-j A R_{\mathrm{s}}}{k_{\mathrm{B}} T / q}\right)-1\right]-\left[j_{\mathrm{ph}}-\frac{V_{\mathrm{app}}}{R_{\mathrm{p}} A}\right]\right\}
$$

Here $V_{\text {app }}$ stands for the applied voltage, that differs from $V_{F}$ by a series voltage due to series resistance $R_{\mathrm{s}}$, and current is reduced by shunt resistance $R_{\mathrm{p}}$, being $A$ the active area. In eq 12 the series resistance voltage drop is explicitly separated from the internal, photovoltaic voltage as $V_{F}=V_{\text {app }}-j A R_{\mathrm{s}}$. Impedance measurements allow us to readily extract resistance effect in series with the recombination mechanism (represented by $C_{\mu}$ and $R_{\text {rec }}$ ) by analyzing the high-frequency part of the response. When the series resistance effect is not severe the analysis proposed in previous sections is still valid as far as chemical capacitance and recombination resistance values are plotted as a function of $V_{F}$ instead of $V_{\text {app }}$. In that case a collapse of $j-V$ curves measured upon different illumination intensities takes place, then signaling the occurrence of a voltage-independent photocurrent as an additional, constant term. ${ }^{21}$

In cases in which transport limitations and limited collection efficiency do matter, the approach implied by eq 12 should be taken with caution because the series resistance potential drop is only a first order approximation of a rather complex picture concerning the Fermi level distribution across the active layer thickness. ${ }^{25}$ In general terms transport mechanisms are coupled with charge storage and recombination in a transmission line circuit model (see below). ${ }^{26,5}$ Only in those cases in which the transport resistance is significantly lower than $R_{\text {rec }}$ the application of the voltage correction in eq 12 is fully justified. This was the case recently reported in which electron transport properties were intentionally perturbed by incorporating fullerene electron traps. ${ }^{27}$ Small amounts ( $\leq 1 \%)$ of PCBM produces an increase in $R_{\mathrm{S}}$ when blended with P3HT:ICBA. Limitation in electron transport occurs as a consequence of the deeper position of PCBM LUMO levels in comparison to ICBA LUMO. While ICBA levels form a sort of transporting band, PCBM molecules can be seen as a trapping site for electrons reducing mobility. Possible effects on $R_{\mathrm{S}}$ caused by outer interfaces were excluded in such a system.

In general terms processes contributing to $R_{\mathrm{S}}$ are voltage-dependent through physical mechanisms originated at different layers or interfaces within the device. Transparent contact layers as indium tin oxide ITO, and carrier transporting interlayers of different kind could increase $R_{\mathrm{s}}$ significantly. Interfaces between the active blend layer and interlayers or metallic contacts may well add more resistance in series because of partial 
energy level alignment which affects optimal interface charge transfer. ${ }^{28}$ Also charge carrier transport within the active layer itself could also be a source for incrementing the series resistance. In addition vertical segregation may influence charge transport owed to blocking effects produced by regions richer in polymer phase in the vicinity of the cell cathode. The overall impact of electronic transport mechanisms is recognized to have dramatic effects when relatively thicker active layer films are used to enhance light harvesting. ${ }^{29}$ While thinner films are able to exhibit almost $100 \%$ conversion of absorbed photons into collected carriers, ${ }^{30}$ thus indicating that transport mechanisms do not limit the achievable photocurrent, ${ }^{21}$ thicker active layer devices suffer from an incomplete collection of photogenerated charges.

For a particular bulk-heterojunction solar cell the current knowledge about the nature and combination of mechanisms contributing as a resistive effect, either due to deficient transport or carrier blocking, is certainly limited. This is particularly true when the kind of voltage separation in eq 12 is no longer valid. A useful approach recently adopted to investigate transport properties in P3HT:PCBM devices has revealed that carriers are collected into the electrodes through the competing mechanisms of diffusion and recombination. Coupling of diffusion-recombination gives rise to equivalent circuits comprising distributed elements, which are usually drawn in terms of transmission line models (see Figure 4a). ${ }^{25-26}$ The simpler cases assume a homogeneous distribution of carriers, which give rise to position-independent distributed circuit elements. The resulting impedance model shown in Figure 4 consists of an equivalent circuit which comprises:

- distributed resistors $r_{\mathrm{t}}=R_{\mathrm{t}} / L$ (being $L$ the active layer thickness), standing for the electron transport. $r_{t}$ is the reciprocal of the electron conductivity $\sigma_{n}$

- distributed chemical capacitance $c_{\mu}=C_{\mu} L$ and

- $r_{\text {rec }}=R_{\text {rec }} / L$ accounting for the electron recombination resistance.

Since holes are regarded as majority carriers (a hole background density exists in the dark) an equipotential line suffices to represent their contribution because of the sufficiently high conductivity of the materials. The impedance can be solved analytically and it corresponds to the expression

$$
Z=\left(\frac{R_{\mathrm{t}} R_{\mathrm{rec}}}{1+i \omega / \omega_{\mathrm{rec}}}\right)^{1 / 2} \operatorname{coth}\left[\left(\frac{\omega_{\mathrm{rec}}}{\omega_{\mathrm{d}}}\right)^{1 / 2}\left(1+\frac{i \omega}{\omega_{\mathrm{rec}}}\right)^{1 / 2}\right]
$$

Here, $\omega_{\mathrm{d}}=D_{n} / L^{2}=1 / R_{\mathrm{t}} C_{\mu}$ is the characteristic frequency of diffusion in a finite Published in Journal of Physical Chemistry Letters 2013, 4, 877-886 
layer ( $D_{n}$ being the electron diffusion coefficient), and $\omega_{\mathrm{rec}}=1 / R_{\mathrm{rec}} C_{\mu}$ is the rate constant for recombination. $\omega$ is the angular frequency, and $i=\sqrt{-1}$. The key parameter which allows establishing the carrier collection efficiency is the diffusion length $L_{n}$ in comparison to the active layer thickness $L . L_{n}$ correlates to the recombination characteristic frequency as $\omega_{\text {rec }}=D_{n} / L_{n}{ }^{2}$. Highly recombining solar cells entails that $L_{n}<<L$, on the contrary efficient collection occurs when $L_{n}>L$. In the conditions of low recombination, $L_{n}>L\left(R_{\mathrm{t}}<<R_{\mathrm{rec}}\right)$, the low-frequency limit of eq 13 reduces to the expression

$$
Z=\frac{1}{3} R_{\mathrm{t}}+\frac{R_{\mathrm{rec}}}{1+i \omega / \omega_{\mathrm{rec}}}
$$

that simply yields a recombination arc (parallel $R_{\mathrm{rec}} C_{\mu}$ circuit) displaced in $R_{\mathrm{t}} / 3$ as illustrated in Figure 4(b). On another hand if we are in conditions of large recombination, $L_{n}<<$, the general impedance of eq 13 becomes the Gerischer impedance,

$$
Z=\left(\frac{R_{\mathrm{t}} R_{\mathrm{rec}}}{1+i \omega / \omega_{\mathrm{rec}}}\right)^{1 / 2}
$$

Figure 4(b) shows the impedance shapes corresponding to the transmission line model of Figure 4(a). The different shapes are obtained by changing only one parameter, the recombination resistance $R_{\text {rec }}$. Curves 1-5 are well approximated by eq 14, while curves 7-8 correspond to Gerischer impedance in eq 15. All impedances show at high frequency a diffusion line of slope 1 , which is implied by eq $13, Z \propto(i \omega)^{-1 / 2}$ at $\omega>>\omega_{\mathrm{d}}$. In the conditions of low recombination $\left(L_{n}>>L\right)$ for curves $1-3$, the $45^{\circ}$ inclined line of diffusion is a minor feature at high frequencies. The impedance is largely dominated by the recombination arc, the second term in eq 14, with the characteristic frequency $\omega_{\mathrm{rec}}$. In contrast, for high recombining cells $L_{n}<<$, the impedance behavior is similar to semi-infinite diffusion, curve 8 . The general case of inhomogeneous distributed circuit elements, particularly important in those cases in which $L_{n}<<L$, has also been treated theoretically. ${ }^{25}$

(a)

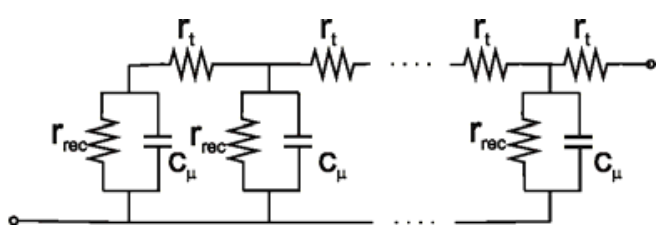

Published in Journal of Physical Chemistry Letters 2013, 4, 877-886 


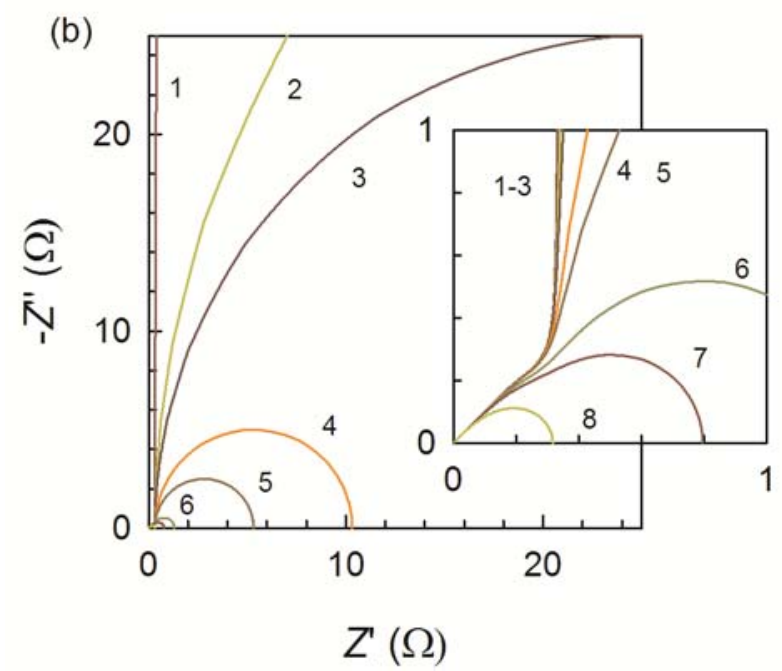

(c)

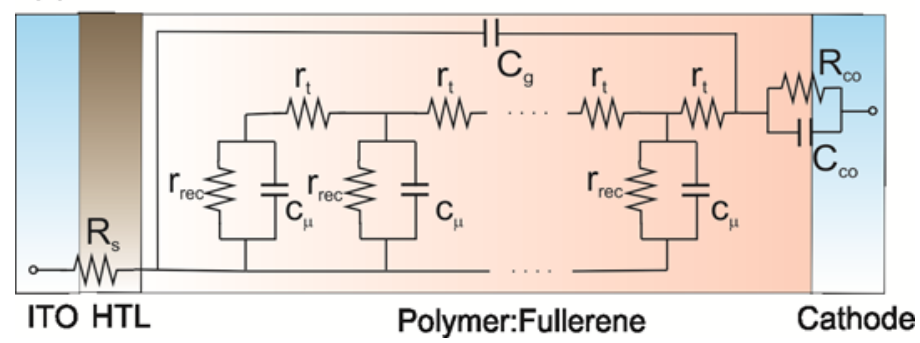

Figure 4. (a) The equivalent circuit (transmission line model) comprising circuit distributed, differential elements related to electron transport $r_{t}$, recombination $r_{\text {rec }}$, chemical capacitance $c_{\mu}$. This circuit accounts for the coupled diffusion-recombination mechanism associated to the active layer response. (b) Simulated impedance spectra for the transmission line in Figure 4a with constant $R_{\mathrm{t}}=1 \Omega$, chemical capacitance $C_{\mu}=10 \mu \mathrm{F}$ and varying $R_{\text {rec }}$. Curves 1-8: $R_{\text {rec }}=10^{4}, 100,50,10,5,1,0.5$ and $0.1 \Omega$. (c) Transmissions line equivalent circuit should be completed in real devices by including geometrical capacitance $C_{\mathrm{g}}$, and series resistance $R_{\mathrm{S}}$. Contact electrical response is modeled by means of $R_{\mathrm{co}} C_{\mathrm{co}}$ parallel subcircuit.

It is known that outer contacts may introduce additional resistive elements particularly when poor energy level alignment occurs between the organic layer and extracting interlayers or contacting metals. IS can also help understanding interfacial phenomena as recently demonstrated for hybrid heterostructures, ${ }^{31}$ and outer contacts. ${ }^{32}$ In Figure 4(c) a contact subcircuit is included in addition to the equivalent circuit accounting for the bulk response. An example of impedance spectra exhibited by 
P3HT:PCBM solar cells under illumination with either extracting (Ca as cathode metal), or blocking $(\mathrm{CaO} / \mathrm{Ca})$ contacts are shown in Figure 5. For blocking contacts formed after metal oxidation huge contact resistances $R_{\text {co }}$ have been observed that becomes much lower for good extracting electrodes. ${ }^{32}$ Such effect is clearly visible in Figure $5(\mathrm{~b})$ as capacitive responses at low frequencies. Interestingly the high-frequency wind exhibits the previously introduced diffusion pattern as $Z \propto(i \omega)^{-1 / 2}$ caused by the transport of electrons within the active layer. For operating solar cells containing $\mathrm{Ca}$ as cathode metal the recombination mechanism dominates the low-frequency response. This occurs for voltages in excess of the maximum power point $\left[V_{\text {app }} \approx 0.5 \mathrm{~V}\right.$ in Figure 5(b)]. By comparing simulated impedance spectra in Figure 4(b), particularly curves 68, with experimental responses in Figure 5(b) at high applied voltages, one can realize that the high-frequency part corresponds to the diffusion mechanism. For the solar cells investigated $^{32}$ it has been observed that $L_{n} \geq L$, an indication of the relatively low transport limitation to carrier extraction. Contacts in real devices operate between ideal extracting and fully blocking electrodes, and even for extracting contacts outer interfaces contribute with an additional, non-negligible, series resistance that accounts for the electron extraction process.

At forward bias voltages approaching $V_{\mathrm{oc}}$ in excess of flat-band conditions diffusion mechanisms lie behind carrier transport. In some circumstances the operation of the solar cell may be affected by the formation of spatially-restricted space charge regions formed by the energetic equilibration between the active layer and the cathode contact (depletion layers). This is assured by the high enough conductivity exhibited by background hole charges resulting from the $p$-doping character of the polymer. However for voltages near short-circuit conditions an extended depletion zone occupies a large part of the active layer thickness. A change in the operation mode would occur between collection favored by depletion zones at low voltages, to diffusion-recombination regime at high voltages. Therefore it would be useful to investigate to what extent charge collection is governed by diffusion near open-circuit and by drift close to shortcircuit conditions, and more importantly if a transition between operating modes can be actually distinguished. Some experiments recently reported, ${ }^{32}$ as the capacitance behavior plotted in Figure 5(b), seem to point to such a change in the operation modes. Capacitance transits from the dielectric, geometrical value $C_{g}$ at low bias related to full-depletion capacitance, to a large chemical capacitance $C_{\mu}$ at voltages approaching $V_{\mathrm{oc}}$ signaling the recombination regime. Additional studies for detailed clarification of 
charge collection mechanisms seem to be required.
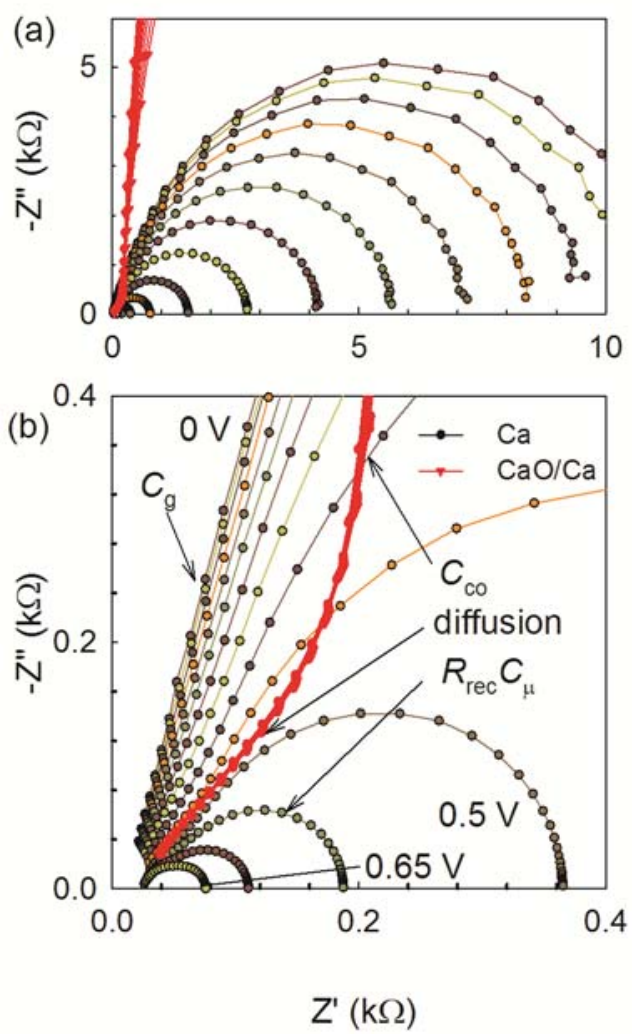

Figure 5. General view (a) and detail (b) of the impedance response from devices using either a good electron collector $(\mathrm{Ca})$ or a blocking layer $(\mathrm{CaO})$, measured under 1 sun illumination by varying the applied voltage between $0 \mathrm{~V}$ and $0.6 \mathrm{~V}$. Impedance spectra plots showing features at low frequencies: recombination $R_{\mathrm{rec}} C_{\mu}$ arc for extracting contacts, and capacitive behavior for blocking contacts $C_{\mathrm{co}}$. For both types of devices diffusion patterns are observed at high frequencies. A transition is observed between spectra dominated by the geometrical capacitance $C_{\mathrm{g}}$ (low bias voltage near shortcircuit voltage) and diffusion patterns at high bias near open circuit voltage.

\section{Associated content}

Supporting Information

Details about materials, device processing, and solar cell performance. This material is available free of charge via the Internet at http://pubs.acs.org.

\section{Acknowledgement}

We thank financial support Ministerio de Educacion y Ciencia (Spain) under project 
HOPE CSD2007-00007 (Consolider-Ingenio 2010), and Generalitat Valenciana (Prometeo/2009/058, and ISIC/2012/008 Institute of Nanotechnologies for Clean Energies).

\section{Author information}

Corresponding author

*E-mail: garciag@,fca.uji.es (G. G.-B.)

\section{Biographies}

Germà Garcia-Belmonte is Professor of Applied Physics (2010) at Universitat Jaume I in Castelló, Spain (Photovoltaic and Optoelectronic Devices Group). He follows researches within the field of Organic Electronics as electronic mechanisms in organic light-emitting diodes, organic photovoltaics, and plastic and thin-film solar cells. Device physics using impedance spectroscopy (including modeling and measuring) is his main subject. (http://www.elp.uji.es/ggb.php)

Antonio Guerrero received a $\mathrm{PhD}$ in organometallic and polymer chemistry at the University of East Anglia (UK) in 2006. He gained understanding in organic semiconductor physics working for Cambridge Display Technology where he learned the design rules to render efficient polymer OLEDs. He currently applies his knowledge at University Jaume I where he fabricates and analyzes OPVs.

Juan Bisquert is a professor of applied physics at Universitat Jaume I de Castelló (http://www.elp.uji.es/jb.php). His main topics of interest are dye- and quantum dotsensitized solar cells, organic solar cells, and solar fuel production. He has developed the application of measurement techniques and physical modeling that relate the device operation with the elementary steps that take place at the nanoscale dimension. 


\section{References}

1.

Green, M. A.; Emery, K.; Hishikawa, Y.; Warta, W.;

Dunlop, E. D., Solar Cell Efficiency Tables (Version 39). Progress in Photovoltaics: Research and Applications 2012, 20, 12-20.

2.

(a) Maurano, A.; Hamilton, R.; Shuttle, C. G.; Ballantyne,

A. M.; Nelson, J.; O'Regan, B.; Zhang, W.; McCulloch, I.; Azimi, H.; Morana, M.; Brabec, C. J.; Durrant, J. R., Recombination Dynamics as a Key Determinant of Open Circuit Voltage in Organic Bulk Heterojunction Solar Cells: a Comparison of Four Different Donor Polymers. Adv. Mat. 2010, 22, 4987-4992; (b) Bisquert, J.; GarciaBelmonte, G., On Voltage, Photovoltage and Photocurrent in Bulk Heterojunction Organic Solar Cells. J. Phys. Chem. Lett. 2011, 2, 1950-1964.

3.

Garcia-Belmonte, G.; Boix, P. P.; Bisquert, J.; Sessolo,

M.; Bolink, H. J., Simultaneous Determination of Carrier Lifetime and Electron Density-of-States in P3HT:PCBM Organic Solar Cells under Illumination by Impedance Spectroscopy. Sol. Energy Mater. Sol. Cells 2010, 94, 366-375.

4. Credgington, D.; Durrant, J. R., Insights from Transient Optoelectronic Analyses on the Open-Circuit Voltage of Organic Solar Cells. J. Phys. Chem. Lett. 2012, 3, 1465-1478.

5 .

Garcia-Belmonte, G.; Munar, A.; Barea, E. M.; Bisquert,

J.; Ugarte, I.; Pacios, R., Charge Carrier Mobility and Lifetime of Organic Bulk Heterojunctions Analyzed by Impedance Spectroscopy. Organic Electronics 2008, 9, 847-851.

6.

(a) Kuwabara, T.; Nakamoto, M.; Kawahara, Y.;

Yamaguchi, T.; Takahasi, K., Characterization of ZnS-Layer-Inserted BulkHeterojunction Organic Solar Cells by ac Impedacne Spectroscopy. J. Appl. Phys. 2009, 105, 124513; (b) Leong, W. L.; Cowan, S. R.; Heeger, A. J., Differential Resistance Analysis of Charge Carrier Losses in Organic Bulk Heterojunction Solar Cells: Observing the Transition from Bimolecular to Trap-Assisted Recombination and Quantifying the Order of Recombination. Advanced Energy Materials 2011, 1, $517-$ 522; (c) Zhang, Y.; Dang, X.-D.; Kim, C.; Nguyen, T.-Q., Charge Recombination on the Fill Factor of Small Molecule Bulk Heterojunction Solar Cells. Advanced Energy Materials 2011, 1, 610-617; (d) Conings, B.; Baeten, L.; Boyen, H.-G.; Spoltore, D.; D'Haen, J.; Grieten, L.; Wagner, P.; Van Bael, M. K.; Manca, J. V., Influence of Interface Morphology onto the Photovoltaic Properties of Nanopatterned ZnO/Poly(3hxylthiophene) Hybrid Solar Cells. An Impedance Spectroscopy Study. Journal of Physical Chemistry C 2011, 115, 16695-16700; (e) Leever, B. J.; Bailey, C. A.; Marks, 
T. J.; Hersam, M. C.; Durstock, M. F., In Situ Characterization of Lifetime and Morphology in Operating Bulk Heterojunction Organic Photovoltaic Devices by Impedance Spectroscopy. Advanced Energy Materials 2012, 2, 120-128; (f) Ecker, B.; Egelhaaf, H.-J.; Steim, R.; Parisi, J.; von Hauff, E., Understanding S- Shaped Current-Voltage Characteristics in Organic Solar Cells Containing a TiOx Interlayer with Impedance Spectroscopy and Equivalent Circuit Analysis. Journal of Physical Chemistry C 2012, 116, 16333-16337; (g) Singh, S. P.; Kumar, C. P.; Sharma, G. D.; Mikroyannidis, J. A.; Singh, M.; Kurchania, P., High-Efficiency Polymer Solar Cells Based on Phenylenevinylene Copolymer with BF2-Azopyrrole Complex and CNPC70BM with Solvent Additive. J. Polym. Sci., Polym. Phys. 2012, 50, 1612-1618; (h)

Perrier, G.; deBettignies, R.; Berson, S.; Lemaître, N.; Guillerez, S., Impedance Spectrometry of Optimized Standard and Inverted P3HT-PCBM Organic Solar Cells. Sol. Energy Mater. Sol. Cells 2012, 101, 210-216.

7.

Garcia-Belmonte, G., Temperature Dependence of Open-

Circuit Voltage in Organic Solar Cells from Generation-Recombination Kinetic Balance. Sol. Energy Mater. Sol. Cells 2010, 94, 2166-2169.

8 .

Blakesley, J. C.; Neher, D., Relationship Between

Energetic Disorder and Open-Circuit Voltage in Bulk Heterojunction Organic Solar Cells. Phys. Rev. B 2011, 84, 075210.

9.

Kaake, L. G.; Barbara, P. F.; Zhu, X.-Y., Intrinsic Charge

Trapping in Organic and Polymeric Semiconductors: a Physical Chemistry Perspective. J. Phys. Chem. Lett. 2010, 1, 628-635.

Fabregat-Santiago, F.; Garcia-Belmonte, G.; Mora-Seró,

I.; Bisquert, J., Characterization of Nanostructured Hybrid and Organic Solar Cells by Impedance Spectroscopy. Phys. Chem. Chem. Phys. 2011, 13, 9083-9118.

11. Bisquert, J., Chemical Capacitance of Nanostructured Semiconductors: its Origin and Significance for Heterogeneous Solar Cells. Phys. Chem. Chem. Phys. 2003, 5, 5360-5364.

(a) Hoshino, S.; Yoshida, M.; Uemura, S.; Kodzasa, T.;

Takada, N.; Kamata, T.; Yase, K., Influence of Moisture on Device Characteristics of Polythiophene-Based Field-Effect Transistors. J. Appl. Phys. 2004, 95, 5088-5093; (b) Abdou, M. S. A.; Orfino, F. P.; Son, Y.; Holdcroft, S., Interaction of Oxygen with Conjugated Polymers: Charge Transfer Complex Formation with Poly(3alkylthiophenes). J. Am. Chem. Soc. 1997, 119, 4518-4524.

13.

(a) Ishii, H.; Hayashi, N.; Ito, E.; Washizu, Y.; Sugi, K.; Kimura, Y.; Niwano, M.; Ouchi, Y.; Seki, K., Kelvin Probe Study of band Bending at Organic Semiconductor/Metal Interfaces: Examination of Fermi level Alignment. Phys. 
Stat. Sol. (a) 2004, 201, 1075-1094; (b) Gregg, B. A.; Liang, Z., Compensating Poly(3hexylthiophene) Reveals Its Doping Density and Its Strong Exciton Quenching by Free Carriers. Adv. Mat. 2012, 24, 3258-3262; (c) Li, J. V.; Nardes, A. M.; Liang, Z.; Shaheen, S. E.; Gregg, B. A.; Levi, D. H., Simultaneous Measurement of Carrier Density and Mobility of Organic Semiconductors Using Capacitance Techniques. Organic Electronics 2011, 12, 1879-1885; (d) Lee, J.; Kong, J.; Kim, H.; Kang, S.-O.; Lee, K., Direct Observation of Internal Potential Distributions in a Bulk Heterojunction Solar Cell. Appl. Phys. Lett. 2011, 99, 243301.

14.

Bisquert, J.; Cahen, D.; Hodes, G.; Rühle, S.; Zaban, A., Physical Chemical Principles of Photovoltaic Conversion with Nanoparticulate, Mesoporous Dye-Sensitized Solar Cells. J. Phys. Chem. B 2004, 108, 8106-8118.

15.

Garcia-Belmonte, G.; Boix, P. P.; Bisquert, J.; Lenes, M.;

Bolink, H. J.; La Rosa, A.; Filippone, S.; Martín, N., Influence of the Intermediate Density-of-States Occupancy on Open-Circuit Voltage of Bulk Heterojunction Solar Cells with Different Fullerene Acceptors. J. Phys. Chem. Lett. 2010, 1, 2566-2571.

16.

Guerrero, A.; Marchesi, L. F.; Boix, P. P.; Bisquert, J.; Garcia-Belmonte, G., Recombination in Organic Bulk Heterojunction Solar Cells: Small Dependence of Interfacial Charge Transfer Kinetics on Fullerene Affinity. J. Phys. Chem. Lett. 2012, 3, 1386-1392.

17.

Boix, P. P.; Ajuria, J.; Etxebarria, I.; Pacios, R.; Garcia-

Belmonte, G.; Bisquert, J., Role of ZnO Electron-Selective Layers in Regular and Inverted Bulk Heterojunction Solar Cells. J. Phys. Chem. Lett. 2011, 2, 407-411.

18.

Wienk, M. M.; Turbiez, M.; Gilot, J.; Janssen, R. A. J., Narrow-Bandgap Diketo-pyrrolo-pyrrole Polymer Solar Cells: the Effect of Processing on the Performance. Adv. Mat. 2008, 20, 2556-2560.

Boix, P. P.; Wienk, M. M.; Janssen, R. A. J.; Garcia-

Belmonte, G., Open-Circuit Voltage Limitation in Low-Bandgap DiketopyrrolopyrroleBased Polymer Solar Cells Processed from Different Solvents. Journal of Physical Chemistry C 2011, 115, 15075-15080.

20.

Garcia-Belmonte, G., Recombination Flux in Bulk

Heterojunction Polymer:Fullerene Solar Cells:Effect of Energy Disorder on Ideality Factor. Solid-St. Electron. 2013, 79, 201-205.

Boix, P. P.; Guerrero, A.; Marchesi, L. F.; Garcia-

Belmonte, G.; Bisquert, J., Current-Voltage Characteristics of Bulk Heterojunction Organic Solar Cells: Connection Between Light and Dark Curves. Advanced Energy Materials 2011, 1, 1073-1078.

22.

Raga, S. R.; Barea, E. M.; Fabregat-Santiago, F., Analysis

Published in Journal of Physical Chemistry Letters 2013, 4, 877-886 
of the Origin of Open Circuit Voltage in Dye Solar Cells. J. Phys. Chem. Lett. 2012, 3, 1629-1634.

23.

(a) Sze, S. M., Physics of Semiconductor Devices. 2nd ed.; John Wiley \& Sons: New York, 1981; (b) Enwin, P.; Thompson, M. E., Elucidating the Interplay Between Dark Current Coupling and Open Circuit Voltage in Organic Photovoltaics. Appl. Phys. Lett. 2011, 98, 223305; (c) Yamamoto, S.; Orimo, A.; Ohkita, H.; Benten, H.; Ito, S., Molecular Understanding of the Open-Circuit Voltage of Polymer:Fullerene Solar Cells. Advanced Energy Materials 2012, 2, 229-237.

24.

Huang, Y.-C.; Tsao, C.-S.; Chuang, C.-M.; Lee, C.-H.; Hsu, F.-H.; Cha, H.-C.; Chen, C.-Y.; Tsung-Han Lin; Chun-Jen Su; Jeng, U.-S.; Su, W.F., Small- and Wide-Angle X-ray Scattering Characterization of Bulk Heterojunction Polymer Solar Cells with Different Fullerene Derivatives. Journal of Physical Chemistry C 2012, 116, 10238-10244.

25.

Pitarch, A.; Garcia-Belmonte, G.; Mora-Seró, I.; Bisquert, J., Electrochemical Impedance Spectra for the Complete Equivalent Circuit of Diffusion and Reaction under Steady-State Recombination Current. Phys. Chem. Chem. Phys. 2004, 6, 2983-2988.

26.

Bisquert, J., Theory of the Impedance of Electron Diffusion and Recombination in a Thin Layer. J. Phys. Chem. B 2002, 106, 325-333.

27.

Guerrero, A.; Ripolles-Sanchis, T.; Boix, P. P.; Garcia-

Belmonte, G., Series Resistance in Organic Bulk-Heterojunction Solar Devices:Modulating Carrier Transport with Fullerene Electron Traps. Organic Electronics 2012, 13, 2326-2332.

28.

(a) Hwang, J.; Wan, A.; Kahn, A., Energetics of MetalOrganic Interfaces: New Experiments and Assessment of the Field. Mater. Sci. Eng. $R$ 2009, 64, 1-31; (b) Ratcliff, E. L.; Zacher, B.; Armstrong, N. R., Selective Interlayers and Contacts in Organic Photovoltaic Cells. J. Phys. Chem. Lett. 2011, 2, 1337-1350; (c) Guerrero, A.; Marchesi, L. F.; Boix, P. P.; Ruiz-Raga, S.; Ripolles-Sanchis, T.; Garcia-Belmonte, G.; Bisquert, J., How the Charge-Neutrality Level of Interface States Controls Energy Level Alignment in Cathode Contacts of Organic Bulk-Heterojunction Solar Cells. ACS Nano 2012, 6, 3453-3460.

29.

Cowan, S. R.; Banerji, N.; Leong, W. L.; Heeger, A. J., Charge Formation, Recombination, and Sweep-Out Dynamics in Organic Solar Cells. Advanced Functional Materials 2012, 22, 1116-1128.

30.

Park, S. H.; Roy, A.; Beaupré, S.; Cho, S.; Coates, N.; Moon, J. S.; Moses, D.; Leclerc, M.; Lee, K.; Heeger, A. J., Bulk Heterojunction Solar Cells with Internal Quantum Efficiency Approaching 100\%. Nature Photonics 2009, 3, 
$297-302$.

31.

(a) Zeng, T.-W.; Ho, C.-C.; Tu, Y.-C.; Tu, G.-Y.; Wang, L.-Y.; Su, W.-F., Correlating Interface Heterostructure, Charge Recombination, and Device Efficiency of Poly(3-hexyl thiophene)/TiO2 Nanorod Solar Cell. Langmuir 2011, 27, 15255-15260; (b) Zeng, T.-W.; Liu, I.-S.; Hsu, F.-C.; Huang, K.-T.; Liao, H.C.; Su, W.-F., Effects of Bifunctional Linker on the Performance of P3HT/CdSe Quantum Dot-Linker-ZnO Nanocolumn Photovoltaic Device. Optics Express 2010, 18, A357-A365.

32.

Ripolles-Sanchis, T.; Guerrero, A.; Bisquert, J.; GarciaBelmonte, G., Diffusion-Recombination Determines Collected Current and Voltage in Polymer:Fullerene Solar Cells. Journal of Physical Chemistry C 2012, 116, $16925-16933$. 


\section{Quotes to highlight in paper}

The chemical capacitance measurement is able to directly monitor the energy position of the relevant DOS actually influencing the final value of the open-circuit voltage in complete cells.

Recombination properties can be discussed by observing the variation of $R_{\text {rec }}$ with voltage or by comparing samples with different morphologies and energetics.

IS is able to capture essential contributions to the open-circuit voltage in a manner easily implementable into industrial processing lines, and establishes a benchmark for the performance comparison of a set of different cells.

TOC

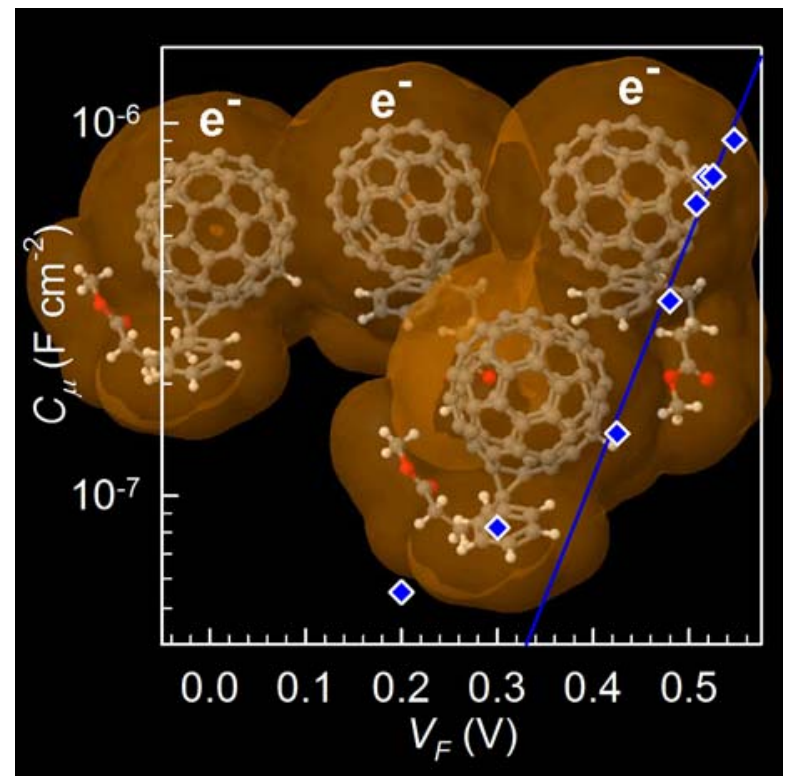

